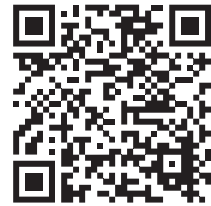

\title{
Riesgo suicida en estudiantes del área de la salud en periodo de pandemia por la COVID-19. Estudio transversal
}

\author{
Suicide risk in health care students during the COVID-19 \\ pandemic period. Cross-sectional study \\ Itzel Betzabeth Sanabria-Landeros, ${ }^{*}$ David Luna, ${ }^{\ddagger}$ Carmen Sánchez-Sánchez, \\ Fernando Meneses-González, ${ }^{\ddagger}$ Miguel Ángel Lezana-Fernández, ${ }^{\ddagger}$ \\ Belinda de la Peña-León,* María Susana González-Velázquez,* Sandro Méndez-García*
}

\begin{abstract}
* Facultad de Estudios Superiores Zaragoza, Universidad Nacional Autónoma de México, Ciudad de México. * Comisión Nacional de Arbitraje Médico, Dirección de Investigación. Ciudad de México.

${ }^{\S}$ Facultad de Enfermería y Fisioterapia. Universidad de Salamanca. España.
\end{abstract}

Correspondencia: IBSL, betzabethlanderos@ gmail.com

Conflicto de intereses: Los autores declaran no tener ningún conflicto de intereses.

Citar como: SanabriaLanderos IB, Luna D, Sánchez-Sánchez C, Meneses-González F, Lezana-Fernández MÁ, De la Peña-León B et al. Riesgo suicida en estudiantes del área de la salud en periodo de pandemia por la COVID-19. Estudio transversal. Rev CONAMED. 2021; 26(4): 182-191. https://dx.doi. org/10.35366/102506

Financiamiento: Ninguno.

Recibido: 18/10/2021. Aceptado: 25/10/2021.

\section{RESUMEN}

Introducción: Debido a la contingencia sanitaria ocasionada por el virus SARS-CoV-2, los estudiantes universitarios del área de la salud están incrementando sus niveles de ansiedad, estrés, depresión y desesperanza, mismos que elevan el riesgo suicida. Objetivo: Determinar en estudiantes del área de la salud en periodo de pandemia por la COVID-19 el nivel de ansiedad, estrés, depresión, desesperanza y riesgo suicida y conocer su asociación. También, identificar variables predictoras de riesgo suicida. Material y métodos: Estudio transversal, comparativo y ex post facto. Participaron 394 estudiantes del área de la salud, 196 mexicanos y 196 españoles. Respondieron una batería en línea que incluía un cuestionario de datos sociodemográficos, académicos y relacionados con la COVID-19, escala de riesgo suicida de Plutchik, escala de depresión, ansiedad y estrés y el índice de desesperanza de Beck. Resultados: En un nivel de moderado a extremadamente severo 66.1\% presentaron ansiedad, 54\% estrés, 49.7\% depresión, 16.8\% tuvieron desesperanza y $69.4 \%$ mostraron riesgo suicida de medio a alto. Ser mexicano, presentar desesperanza, nivel de moderado a extremadamente severo de ansiedad y depresión, y diagnóstico de COVID-19 de algún familiar y/o amigo son predictores del riesgo suicida. Conclusiones: El porcentaje de estudiantes con riesgo suicida es elevado, por lo cual es indispensable se realicen intervenciones de prevención y atención en dicha población. Este trabajo identifica factores que deben ser atendidos para disminuir el riesgo suicida.

Palabras clave: COVID-19, suicidio, ansiedad, depresión, estrés, desesperanza. 
risk prediction factors of suicide. Conclusion: There is a high percentage of students with depression, anxiety, stress and at risk of suicide. This identified different risk factors that should be prevented and addressed to reduce suicide in university students.

Keywords: COVID-19, suicide, anxiety, depression, stress, hopelessness.

\section{INTRODUCCIÓN}

En diciembre de 2019 en la ciudad de Wuhan, China se reportó un nuevo tipo de coronavirus denominado SARS-CoV-2 que ocasiona la Corona Virus Disease 2019 o COVID-19 por sus siglas en inglés.' Por su rápida transmisibilidad, esta enfermedad fue declarada pandemia por la Organización Mundial de la Salud (OMS) en marzo de 2020. ${ }^{2}$ Actualmente, se estiman 242,000,000 de casos confirmados en el mundo y al menos 4,900,000 de fallecidos. ${ }^{3} \mathrm{Su}$ cuadro clínico leve se manifiesta por fiebre, tos, fatiga, anosmia, ageusia, congestión nasal y diarrea; el moderado presenta neumonía y el grave síndrome de dificultad respiratoria aguda (SDRA), pudiendo pasar a estado crítico manifestándose con un choque séptico. ${ }^{4}$ Actualmente se estiman 74.68 millones de casos confirmados en Europa, de los cuales España acumula 4.99 millones con un total de 87,102 defunciones. ${ }^{5}$ En América se registran 92.52 millones de casos confirmados, de los cuales 45.83 se encuentran en Latinoamérica. ${ }^{6}$ En México se contabilizan 3.77 millones de casos confirmados y 299,081 defunciones. ${ }^{7}$

Para reducir la alta tasa de contagios, los gobiernos de diversos países tomaron medidas sanitarias, entre las cuales se incluía el confinamiento. ${ }^{8}$ Pese a la reducción de contagios, esto también impactó negativamente en la salud mental de la población, ${ }^{9}$ aumentando los niveles de ansiedad, depresión y desesperanza..$^{10}$ Estudios recientes en población de México ${ }^{17}$ y España. ${ }^{12}$ indican una prevalencia de alrededor de $40 \%$ de ansiedad y de 30 a $47 \%$ de depresión. Condiciones que incrementan el riesgo suicida en población general y en personas que presentan trastornos psiquiátricos, emplean sustancias nocivas e incluso en profesionales de la salud. 13,14 En estos últimos debido a las excesivas jornadas de trabajo, fatiga, riesgo de infección y falta de equipo de protección personal, que exacerban condiciones psicopatológicas preexistentes. ${ }^{9}$

Una población de riesgo poco reconocida son los estudiantes universitarios, quienes previo a la pandemia ya presentaban alta prevalencia de psicopatologías, incluyendo depresión y ansiedad. Además, en esta población, son los estudiantes del área de la salud quienes se ven más afectados. ${ }^{15}$ Datos previos a la pandemia indicaban una prevalencia de 73 a $79 \%$ de ansiedad ${ }^{16,17}$ y alrededor de 23\% 18,19 de depresión, con riesgo suicida entre 11 y 30\%. ${ }^{20}$ Datos actuales de estudios realizados en China, ${ }^{21}$ España ${ }^{22}$ y Blangadesh ${ }^{23}$ indican evidencia de insomnio y niveles elevados de ansiedad, estrés y depresión en esta población, asociados a la pandemia por la COVID-19. Estas condiciones se han desencadenado por el miedo a contagiarse, tener un familiar diagnosticado de SARS-CoV-2, el distanciamiento social, la preocupación por su situación académica y el aprendizaje en línea. ${ }^{24,25}$ Sin embargo, actualmente no hay estudios realizados en población mexicana.

En la era de COVID-19 el riesgo suicida es un tema poco explorado en particular en estudiantes universitarios, pese a que las condiciones sanitarias vigentes y de salud mental pueden potencializar el riesgo de cometer suicidio. ${ }^{10,26} \mathrm{En}$ la literatura se han reportado diversos suicidios en periodo de pandemia por la COVID-19, entre ellos un estudiante chino en Arabia Saudita saltó de un tercer piso del hospital donde se encontraba aislado por sospecha de diagnóstico de SARS-CoV-2 y una joven de 19 años en la Gran Bretaña lo hizo por miedo al encierro producto del confinamiento. ${ }^{10}$

En este contexto, el objetivo de este estudio fue determinar en estudiantes mexicanos y españoles del área de la salud en periodo de pandemia por la COVID-19, el nivel de ansiedad, estrés, depresión, desesperanza y riesgo suicida; igualmente, evaluar la asociación entre estas variables e identificar predictores de riesgo suicida. La población de estudio se eligió debido a que los estudiantes españoles se asistían a clases presenciales, mientras que los estudiantes mexicanos se enfrentaban a un aprendizaje en línea, mismo que puede tener más afectaciones en la salud mental. ${ }^{24}$ 


\section{MATERIAL Y MÉTODOS}

Diseño de estudio: estudio transversal comparativo y ex post facto. ${ }^{27}$

Muestra: se reclutó a los participantes a través de una técnica de muestreo no probabilístico por conveniencia. El universo de estudio estuvo conformado por estudiantes de las carreras de enfermería, medicina, psicología y odontología inscritos en la Facultad de Estudios Superiores Zaragoza, México y se anexaron las carreras de Fisioterapia y Terapia Ocupacional para los estudiantes inscritos en la Universidad de Salamanca, España. Los criterios de inclusión fueron participación voluntaria y tener 18 años o más. Se excluyeron los datos de participantes que cerraron la batería en línea antes de concluir su respuesta.

Instrumentos: cuestionario con datos sociodemográficos y académicos. Se recopiló información sobre edad, sexo, estado civil, carrera, año cursado y asistencia a tratamiento psicológico.

Cuestionario COVID-19. Identificó si el participante tenía temor a contagiarse y si algún familiar y/o amigo se ha contagiado o fallecido a causa de la COVID-19.

Escala de riesgo suicida de Plutchik ${ }^{28}$ validada en población mexicana. ${ }^{29}$ Comprende 15 preguntas dicotómicas (No: O, Sí: 1) El valor por reactivo se suma y un puntaje > seis indica presencia de riesgo suicida. Explica $49.7 \%$ de la varianza con un $\alpha$ Cronbach $=0.74 .{ }^{29}$

Escala de depresión, ansiedad y estrés (DASS-21) versión en español. ${ }^{30}$ Se compone de 21 reactivos Likert (de cero a tres puntos) con tres factores de siete reactivos cada uno: depresión, ansiedad y estrés. El puntaje obtenido por factor se multiplica por dos y se considera depresión normal (de cero a nueve), baja (10-13), moderada (14-15), severa (21-27) y extremadamente severa ( $\geq 28$ ); ansiedad normal (de cero a siete), baja (de ocho a nueve), moderada (1014), severa (15-19) y extremadamente severa ( $\geq 20)$; y estrés normal (de cero a 14), bajo (15-18), moderado (19-25), severo (26-33) y extremadamente severo ( $\geq$ 34). ${ }^{31}$ La DASS-21 tiene un $\alpha$ Cronbach $=0.96$.

Índice de desesperanza de Beck validada con población mexicana. ${ }^{32}$ Comprende 20 reactivos dicotómicos (uno o cero). El valor por reactivo se suma y se interpreta de cero a tres normal o asintomático; de cuatro a ocho leve; de nueve a 14 moderado y
15-20 severo. Un total > nueve se considera predictor de conducta suicida. ${ }^{33}$ Tiene un $\Omega=0.89$.

\section{Procedimiento}

La recolección de información se realizó mediante la difusión de una batería en línea con un vínculo o un código QR. Fue respondida con una técnica de autorreporte. Se difundió de enero a abril en población mexicana y de mayo a agosto en población española de 2021. Previo a la difusión de la batería, en la población española se realizó una adaptación cultural donde se modificaron algunas palabras de los reactivos a fin de asegurar su buena comprensión (p. ej. cubículo se sustituyó por despacho). Los cambios hechos fueron revisados y validados por una investigadora nativa castellana.

\section{Análisis de resultados}

Se emplearon frecuenciasy porcentajes para evaluar los datos sociodemográficos y de COVID-19 así como para determinar la prevalencia del nivel de ansiedad, estrés, depresión, desesperanza y riesgo suicida. Para comparar los resultados de ambas nacionalidades y evaluar la asociación entre variables se empleó la prueba $\chi^{2}$ de independencia. Con un resultado significativo se calcularon los residuos estandarizados de Pearson como prueba post hoc y la $\vee$ de Cramer como indicador del tamaño del efecto. La fuerza de asociación entre variables se interpretó trivial con valores absolutos menores de 0.10, 0.11-0.29 baja, 0.30-0.49 media y $\geq 0.50$ alta. ${ }^{34}$ Se estimó un modelo de regresión logística basada en el estadístico de Wald que consideró como variable de respuesta la presencia de riesgo suicida en los participantes y como variables predictoras el sexo, nacionalidad, asistencia a terapia psicológica, nivel de ansiedad, depresión, estrés, desesperanza y las preguntas del cuestionario COVID-19. Se estimó el intervalo a 95\% de los coeficientes obtenidos. El modelo se validó por evaluar la hipótesis nula (prueba ómnibus) y su bondad de ajuste (Hosmer-Lemeshow). Se calculó el porcentaje de clasificación de casos y la $R^{2}$ de Nagelkerke. ${ }^{35}$ Se empleó el programa SPSS v.20 y un resultado se consideró significativo con $p \leq 0.05$.

Consideraciones éticas: el Comité de Investigación y la Comisión de Bioética del Comité de 
Investigación de la Facultad de Estudios Superiores Zaragoza aprobaron esta investigación. La participación fue voluntaria y anónima y no se entregó ningún incentivo a cambio.

\section{RESULTADOS}

Participaron 392 estudiantes, 196 mexicanos y 196 españoles, edad media 21 años (DE = 3.61). La Tabla 7 muestra los datos sociodemográficos y académicos de los participantes en general y por nacionalidad. En ambas poblaciones el mayor porcentaje de participantes fueron del sexo femenino, de las carreras de enfermería y psicología, cursaban el primer o segundo año de formación y no acudían a tratamiento psicológico.

La Tabla 2 muestra los resultados del cuestionario COVID-19. El mayor porcentaje de participantes tienen miedo a contagiarse de la COVID-19, aunque sólo un bajo porcentaje ha sido confirmado positivo a esta enfermedad. De los participantes, 83.2\% tiene un amigo y/o familiar que ha sido diagnosticado de la COVID-19 y 26.5\% han sufrido la muerte de alguno o ambos. Ninguna de estas condiciones se asoció a la presencia de riesgo suicida ( $p>0.05)$. La ausencia de desesperanza se asoció a la presencia de miedo al contagio $\left(\chi^{2}=4.52 ; \mathrm{V}=0.10\right)$ y a la ausencia de diagnóstico positivo $\left(\chi^{2}=7.52 ; \vee V=0.13\right)$; la presencia de desesperanza se asoció a la ausencia de miedo al contagio y con un diagnóstico positivo $(p<0.01)$. La fuerza de asociación fue baja en todos los casos. Se detectó la mayor frecuencia de diagnóstico positivo en población española, mientras que en la población mexicana este diagnóstico se identificó con más frecuencia en familiares y amigos, los cuales igualmente presentaron mayor frecuencia de fallecimiento. Ninguna otra comparación resultó significativa ( $p>0.05)$.

En la Tabla 3 se muestra la prevalencia del nivel de ansiedad, estrés, depresión, desesperanza y riesgo suicida así como la asociación de esta última con las primeras. Esta asociación se evaluó por integrar las categorías «sin riesgo suicida» (nivel nulo a bajo) $\mathrm{y}$ «con riesgo suicida» (nivel medio a alto). De nivel moderado a extremadamente severo $66.1 \%$ de los participantes reportó ansiedad, 54\% estrés y 49.7\% depresión. Sólo un bajo porcentaje de participantes presentó desesperanza. La ansiedad, depresión y desesperanza se asociaron con riesgo suicida.
La fuerza de asociación osciló entre media y alta. La comparación entre nacionalidades indicó que en españoles hubo mayor frecuencia de estrés moderado, mientras que en mexicanos el estrés y la depresión en nivel normal fue más frecuente. Ninguna otra comparación resultó significativa ( $\mathrm{P}$ $>0.05$ ).

La Tabla 4 muestra la regresión logística que identificó como factores predictores de riesgo suicida «ser mexicano», presencia de «desesperanza», nivel de moderado a extremadamente severo de «ansiedad» y «depresión» así como el «diagnóstico positivo de COVID-19 para algún familiar y/o amigo». El modelo fue significativo $\left(\chi^{2}=139.96, p<\right.$ $0.001)$, con adecuado ajuste $\left(\chi^{2}=5.44, p=0.70\right)$, explicó $41.9 \%$ de la varianza y clasificó correctamente $76.5 \%$ de los casos.

\begin{tabular}{lcc}
\multicolumn{3}{c}{ Tabla 1: Datos sociodemográficos y } \\
académicos de estudiantes universitarios \\
del área de la salud. N = 392.
\end{tabular}


Tabla 2: Resultados del cuestionario COVID-19.

\begin{tabular}{|c|c|c|c|c|c|c|}
\hline & \multicolumn{3}{|c|}{$n(\%)$} & \multirow[b]{2}{*}{$\chi^{2}$} & \multirow[b]{2}{*}{$\mathrm{p}$} & \multirow[b]{2}{*}{ V de Cramer } \\
\hline & Total & Mexicanos & Españoles & & & \\
\hline Miedo de contagio & & & & 1.15 & 0.28 & \\
\hline Sí & $301(76.8)$ & $155(79.1)$ & $146(74.5)$ & & & \\
\hline No & $91(23.2)$ & $41(20.9)$ & $50(25.5)$ & & & \\
\hline Diagnóstico positivo & & & & 20.32 & 0.01 & 0.22 \\
\hline Sí & $55(14.0)$ & $12(6.1)$ & $43(21.9)^{\S}$ & & & \\
\hline No & $337(86.0)$ & $184(93.9)^{5}$ & $153(78.1)$ & & & \\
\hline Diagnóstico positivo en familiares y amigos & & & & 60.54 & 0.01 & 0.39 \\
\hline No & $66(16.8)$ & $46(23.5)^{\S}$ & $20(10.2)$ & & & \\
\hline Sí, a un familiar & $77(19.6)$ & $62(31.6)^{\S}$ & $15(7.7)$ & & & \\
\hline Sí, a un amigo & $61(15.6)$ & $20(10.2)$ & $41(20.9)^{\S}$ & & & \\
\hline Sí, a familiares y amigos & $188(48.0)$ & $68(34.7)$ & $120(61.2)^{\S}$ & & & \\
\hline Fallecimiento de familiares y amigos & & & & 22.16 & 0.01 & 0.23 \\
\hline No & $288(73.5)$ & $126(64.3)$ & $162(82.7)^{\S}$ & & & \\
\hline Sí, a un familiar & 64 (16.3) & $39(19.9)$ & $25(12.8)$ & & & \\
\hline Sí, a un amigo & $20(5.1)$ & $13(6.6)$ & $7(3.6)$ & & & \\
\hline Sí, a familiares y amigos & $20(5.1)$ & $18(9.2)^{\S}$ & $2(1.0)$ & & & \\
\hline
\end{tabular}

$\S=$ Frecuencia mayor a la esperada por azar.

\section{DISCUSIÓN}

Se detectó una alta prevalencia de ansiedad, depresión y estrés. Un bajo porcentaje de estudiantes presentó desesperanza, aunque se identificó que $67.6 \%$ tiene un riesgo suicida de medio a alto. La ansiedad, depresión y desesperanza se asocian con riesgo suicida. Los predictores de riesgo suicida, en orden de importancia, fueron la presencia de «desesperanza» de moderado a extremadamente severo, presencia de «depresión» moderada a extremadamente severa que algún «familiar y/o amigo fuera diagnosticado de COVID-19», presencia de «ansiedad» moderada a extremadamente severa y «ser mexicano». El diagnóstico positivo a la COVID-19 fue más frecuente en españoles, mientras que una mayor frecuencia de fallecimientos de familiares y amigos fue detectada en mexicanos.

El mayor porcentaje de diagnóstico positivo de COVID-19 y el nivel moderado de estrés en estudiantes españoles pudo ser debido a que éstos asisten a clases presenciales, a diferencia de los mexicanos que se encuentran en aprendizaje a distancia. La mayor frecuencia de fallecimientos de familiares y amigos en estudiantes mexicanos podría estar relacionada con el manejo de la pandemia que las autoridades sanitarias han llevado a cabo. A favor de esta interpretación, datos actuales indican una alta tasa de mortalidad en México ${ }^{36}$ a diferencia de otros países, incluyendo España.

El nivel de ansiedad, estrés y depresión es semejante al reportado en otros estudios realizados en periodo de pandemia, 21,22,25,37 cuyo rango va de 18.1 a $67.55 \% .22,25$ Es semejante también a valores descritos prepandemia. ${ }^{19,38}$ La semejanza en prevalencias de ansiedad y depresión durante y previo a la pandemia cuestiona que la contingencia misma sea un factor causal de éstas, aunque los datos actuales no permiten aceptar o rechazar esta afirmación. Sin embargo, la prevalencia de estrés prepandemia fue menor ${ }^{38}$ que la reportada en periodo de pandemia por Odriozola y colaboradores ${ }^{22}$ y en el presente estudio esto indica que los niveles de estrés sí aumentaron en este 


\begin{tabular}{|c|c|c|c|c|c|c|c|c|c|c|c|}
\hline & \multicolumn{3}{|c|}{ n (\%) } & \multirow[b]{2}{*}{$\chi^{2}$} & \multirow[b]{2}{*}{$p$} & \multirow[b]{2}{*}{ V } & \multicolumn{2}{|c|}{$n(\%)$} & \multirow[b]{2}{*}{$\chi^{2}$} & \multirow[b]{2}{*}{$\mathrm{p}$} & \multirow[b]{2}{*}{ V de Crame } \\
\hline & Total & Con riesgo & Sin riesgo & & & & Mexicanos & Españoles & & & \\
\hline Ansiedad & & & & 80.78 & 0.01 & 0.45 & & & 4.44 & 0.34 & \\
\hline Normal & $121(30.9)$ & $51(13.0)$ & $70(17.9)^{\S}$ & & & & $110(28.1)$ & 93 (23.7) & & & \\
\hline No severo & $12(3.1)$ & $5(1.3)$ & 7 (1.8) & & & & $18(4.6)$ & $18(4.6)$ & & & \\
\hline Moderado & 70 (17.9) & $42(10.7)$ & $28(7.1)$ & & & & $39(9.9)$ & $48(12.2)$ & & & \\
\hline Severo & $36(9.2)$ & $28(7.1)$ & $8(2)$ & & & & $25(6.4)$ & $28(7.1)$ & & & \\
\hline $\begin{array}{l}\text { Extremadamente } \\
\text { severo }\end{array}$ & $153(39)$ & $139(35.5)^{\S}$ & $14(3.6)$ & & & & $4(1.0)$ & $9(2.3)$ & & & \\
\hline Estrés & Dis & & & 69.24 & 0.01 & 0.42 & & & 11.28 & 0.04 & 0.17 \\
\hline Normal & $127(32.4)$ & $56(14.3)$ & $71(18.1)^{\S}$ & & & & $156(39.8)^{\S}$ & $129(32.9)$ & & & \\
\hline No severo & $53(13.5)$ & $31(7.9)$ & $22(5.6)$ & & & & $32(8.2)$ & 45 (11.5) & & & \\
\hline Moderado & $58(14.8)$ & $40(10.2)$ & $18(4.6)$ & & & & $8(2.0)$ & $22(5.6)^{5}$ & & & \\
\hline Severo & $97(24.7)$ & $84(21.4)$ & $13(3.3)$ & & & & - & - & & & \\
\hline $\begin{array}{l}\text { Extremadamente } \\
\text { severo }\end{array}$ & $57(14.5)$ & $54(13.8)^{\S}$ & $2(0.8)$ & & & & - & - & & & \\
\hline Depresión & $\sum_{-1}$ & & & 103 & 0.01 & 0.51 & & & & & \\
\hline Normal & $154(39.3)$ & $62(15.8)$ & $92(23.5)^{\S}$ & & & & $144(36.7)^{\S}$ & 117 (29.8) & 8.51 & 0.03 & 0.14 \\
\hline No severo & $43(11.0)$ & $26(6.6)$ & $17(4.3)$ & & & & $25(6.4)$ & $37(9.4)$ & & & \\
\hline Moderado & 77 (19.6) & $66(16.8)^{\S}$ & $11(2.8)$ & & & & $24(6.1)$ & $36(9.2)$ & & & \\
\hline Severo & $=49(12.5)$ & $45(11.5)^{\S}$ & $4(1)$ & & & & $3(0.8)$ & $6(1.5)$ & & & \\
\hline $\begin{array}{l}\text { Extremadamente } \\
\text { severo }\end{array}$ & $69(17.6)$ & $66(16.8)^{\S}$ & $3(0.8)$ & & & & - & - & & & \\
\hline Desesperanza & & & & 38.38 & 0.01 & 0.31 & & & 3.50 & 0.06 & \\
\hline $\operatorname{Sin}$ & $163(83.2)^{\S}$ & $187(47.7)$ & $124(31.6)$ & & & & $163(41.6)$ & $148(37.8)$ & & & \\
\hline Con & $33(16.8)$ & 78 (19.9) & $3(0.8)^{5}$ & & & & $33(8.4)$ & $48(12.2)$ & & & \\
\hline Riesgo suicida & & & & & & & & & 2.98 & 0.39 & \\
\hline Sin riesgo & $67(17.1)$ & & & & & & $36(9.2)$ & $31(7.9)$ & & & \\
\hline Bajo & $60(15.3)$ & & & & & & $36(9.2)$ & $36(9.2)$ & & & \\
\hline Medio & $159(40.6)$ & & & & & & 78 (19.9) & 78 (19.9) & & & \\
\hline Alto & $106(27.0)$ & & & & & & 51 (13.0) & 51 (13.0) & & & \\
\hline
\end{tabular}

${ }^{s}=$ Frecuencia mayor a la esperada por azar. 
Tabla 4: Factores predictores de riesgo suicida en estudiantes universitarios del área de la salud.

\begin{tabular}{|c|c|c|c|c|}
\hline & $\begin{array}{l}\text { Coeficiente de } \\
\text { regresión }\end{array}$ & Odds Ratio & IC 95\% & $p$ \\
\hline \multicolumn{5}{|l|}{ Nacionalidad } \\
\hline Mexicana & 0.65 & 1.92 & 7.08 a 3.41 & 0.026 \\
\hline \multicolumn{5}{|l|}{ DASS-21 ansiedad } \\
\hline Moderado a extremadamente severo & 0.82 & 2.27 & 1.27 a 4.07 & 0.060 \\
\hline \multicolumn{5}{|l|}{ DASS-21 depresión } \\
\hline Moderado a extremadamente severo & 1.96 & 7.12 & 3.7 a 13.68 & 0.010 \\
\hline \multicolumn{5}{|l|}{ IDB } \\
\hline Con desesperanza & 2.25 & 9.51 & 2.73 a 33.13 & 0.010 \\
\hline \multicolumn{5}{|l|}{ Diagnóstico COVID-19 } \\
\hline Diagnóstico personal & -0.048 & 0.95 & 0.41 a 2.21 & 0.910 \\
\hline Diagnóstico a familiares y/o amigos & 0.91 & 2.49 & 0.98 a 6.35 & 0.055 \\
\hline Muerte de familiares y/o amigos & -0.175 & 0.83 & 0.39 a 1.78 & 0.640 \\
\hline
\end{tabular}

$\mathrm{R}$ = Categoría de referencia. $\mathrm{p}=$ nivel de significancia, DASS-21 = escala de depresión, ansiedad y estrés, IDB = Índice de desesperanza de Beck.

periodo probablemente debido a la contingencia sanitaria.

Con estudiantes universitarios durante periodo de pandemia, Santana y su equipo ${ }^{26}$ reportaron la presencia de desesperanza. Los datos aquí mostrados confirman este hecho y lo extienden por cuantificar su prevalencia. Estos datos son también consistentes con datos obtenidos en población general ${ }^{39}$ y con jóvenes adultos. ${ }^{40}$

La alta prevalencia de riesgo suicida detectada en estudiantes del área de la salud rebasa la reportada en muestras semejantes,,$^{41}$ en población genera ${ }^{23,42}$ y en estudiantes adolescentes con intento suicida. ${ }^{43}$ La alta prevalencia de ansiedad, estrés, depresión y riesgo suicida así como la presencia de desesperanza pudo ser debida a que los estudiantes se encuentran en distanciamiento social y con abandono de sus actividades cotidianas, aunado al aprendizaje en línea, ${ }^{24,25}$ además de tener miedo de contraer el virus y de que un familiar muera a causa de COVID-19.4] El amplio rango de prevalencias en estas condiciones pudo ser debido, entre otros factores, al momento de la pandemia en que se realizó el estudio, a los instrumentos empleados para la medición de las condiciones evaluadas y a las características específicas de la población analizada.

Consistente con Rahman y colaboradores, ${ }^{23}$ la ansiedad y la depresión se asociaron con riesgo suicida. Por el contrario, en el presente estudio no se detectó evidencia de asociación entre riesgo suicida y miedo al contagio o muerte de algún familiar. En cambio, se identificó la asociación entre riesgo suicida y desesperanza ${ }^{39}$ y una asociación entre ausencia de desesperanza con miedo al contagio y ausencia de diagnóstico positivo. Lo anterior se puede atribuir a que el miedo al contagio quizás promovió conductas de autocuidado con las que se evitó contraer el virus. La presencia de desesperanza se asoció con la ausencia de miedo y el diagnóstico positivo de COVID-19, quizás debido a que la primera interfirió con la ejecución de conductas de autocuidado y promovió conductas de riesgo. 
El mejor predictor de riesgo suicida fue la «desesperanza» de moderado a extremadamente severo, seguida de «depresión» en nivel moderado a extremadamente severo, que «algún familiar y/o amigo fuera diagnosticado de COVID-19», «ansiedad» en nivel moderado a extremadamente severo y «ser mexicano». Los factores «depresión» y «ansiedad» son consistentes con datos obtenidos en otros estudios, ${ }^{10,26,43}$ mientras que los factores «ser mexicano» y el «diagnóstico positivo a familiares y/o amigos» aportan conocimiento útil para complementar la relación entre la pandemia y el riesgo suicida.

El predictor suicida «ser mexicano» pudo ser debido al aprendizaje en línea, el cual trastorna las actividades cotidianas y sociales de los estudiantes y por la difícil integración familiar reportada por éstos en otros estudios, ${ }^{26}$ a diferencia de los estudiantes españoles, quienes al momento de realizar este estudio acudían a clases presenciales. El diagnóstico positivo a familiares y/o amigos como predictor de riesgo suicida pudo ser debido a la incertidumbre que el participante sintiera sobre la salud y la vida de éstos así como la probabilidad de que este mismo se contagiara de COVID-19.23

Los datos en el presente estudio tienen varias implicaciones, subrayan la necesidad que las entidades educativas tienen respecto al diseño e instrumentación de programas de promoción a la salud, detección temprana y atención de diversas psicopatologías así como de riesgo suicida. Igualmente, ante casos detectados, el compromiso de canalizarlos con un especialista dentro de un programa institucional resalta la necesidad de conducir un mayor número de estudios que indaguen el estado actual del riesgo suicida durante este periodo de pandemia.

Se propone que futuras investigaciones incluyan el estudio de factores protectores del riesgo suicida y que promuevan la salud mental positiva. Esto debido a que por lo general los trabajos realizados se enfocan en psicopatologías, dejando de lado factores promotores del desarrollo y bienestar humanos. Estudiantes de diversas áreas y grupos etarios deberán ser también analizados.

Entre las limitaciones de este estudio se encuentran el tamaño de la muestra y su carácter no aleatorio, lo cual compromete la generalidad de los resultados. Por esto último, las interpretaciones aquí elaboradas deben ser tomadas con cautela. I gualmente, existe ausencia de información de los estudiantes respecto a su situación económica y social, empleo y vacunación COVID-19. Información que podría arrojar datos útiles para futuras investigaciones.

\section{CONCLUSIONES}

Se identificaron altos niveles de ansiedad, depresión, estrés y de riesgo suicida. Si bien la presencia de desesperanza es baja, es el mejor predictor de riesgo suicida seguido de depresión en nivel moderado a extremadamente severo, que algún familiar y/o amigo se encuentre diagnosticado de COVID-19, ansiedad en nivel moderado a extremadamente severo y ser mexicano. Igualmente, en población mexicana se detectó mayor frecuencia de fallecimientos de familiares y amigos, mientras que en población española se identificó mayor frecuencia de diagnóstico positivo propio.

Se recomienda que las entidades académicas instrumenten oportunamente estrategias para identificar psicopatologías que incluyan ansiedad, depresión, estrés, desesperanza y riesgo suicida, así como estrategias de afrontamiento para los estudiantes vulnerables.

\section{AGRADECIMIENTOS}

A la Universidad de Salamanca, España, a la Universidad Nacional Autónoma de México y a la Comisión Nacional de Arbitraje Médico por las facilidades otorgadas para la realización de este estudio.

\section{REFERENCIAS}

1. Li Q, Guan X, Wu P, Wang X, Zhou L, Tong Y et al. Early transmission dynamics in Wuhan, China, of novel coronavirus-infected pneumonia. N Engl J Med. 2020; 382 (13): 1199-1207.

2. Cronología de la respuesta de la OMS a la COVID-19 [Internet]. [Cited 2021 Sep 23]. Available in: https://www. who.int/es/news/item/29-06-2020-covidtimeline

3. COVID Live Update: $243,551,724$ Cases and $4,949,756$ Deaths from the Coronavirus - Worldometer [Internet]. [Cited 2021 Oct 22]. Available in: https://www.worldometers. info/coronavirus/ 
4. Gobierno de México. Guía clínica para el tratamiento de la COVID-19 en México [Internet]. Available in: https:// coronavirus.gob.mx/wp-content/uploads/2021/08/ GuiaTx_COVID19_Consensolnterinstitucional_2021.08.03. pdf

5. EURO_COVID19_Dashboard - PUBLIC [Internet]. [Cited 2021 Oct 22]. Available in: https://who.maps.arcgis.com/ apps/dashboards/ead3c6475654481ca51c248d52ab9c61

6. Panel de control de coronavirus (COVID-19) de la OMS [Internet]. [Cited 2021 Oct 22]. Disponible en: https://covid19. who.int/table

7. COVID-19 Tablero México - CONACYT - CentroGeo - Geolnt - DataLab [Internet]. [Cited 2021 Oct 22]. Disponible en: https://datos.covid-19.conacyt.mx/

8. SANA DISTANCIA COVID-19/Secretaría de Salud | Gobierno | gob.mx [Internet]. [Citado 2021 Oct 17]. Disponible en: https://www.gob.mx/salud/documentos/sana-distancia

9. Nicolini H. Depression and anxiety during COVID-19 pandemic. Cir y Cir. 2020; 88 (5): 542-547.

10. Thakur V, Jain A. COVID2019-suicides: a global psychological pandemic. Brain Behav Immun. 2020; 88: 952-953.

11. Pérez-Cano HJ, Moreno-Murguía MB, Morales-López O, Crow-Buchanan O, English JA, Lozano-Alcázar J et al. Anxiety, depression, and stress in response to the coronavirus disease-19 pandemic. Cir y Cir. 2020; 88 (5): 562-568.

12. Jacques-Aviño C, de Bont J BA. Social conditions and mental health during COVID-19 lockdown among people who do not identify with the man/woman binomial in Spain. PLoS One. 2021; 16 (8): e0256261.

13. Sher L. Psychiatric disorders and suicide in the COVID-19 era. QJM. 2020; 113 (8): 527-528.

14. Moitra M, Rahman M, Collins PY, Gohar F, Weaver M, Kinuthia J et al. Mental health consequences for healthcare workers during the COVID-19 pandemic: a scoping review to draw lessons for LMICs. Front Psychiatry. 2027; 12: 602614.

15. Segura ANA, Eraña RIE, Valeria LGM, Castorena-Ibarr J, López CMV. Analysis of anxiety on early clinical encounters: experiences using clinical simulation in undergraduate students. Educ Medica. 2020; 21 (6): 377-382

16. Inam SN, Sagib AAE. Prevalence of anxiety and depression among medical students of private university. Public Health. 2003; 53 (2): 44-47.

17. Aboalshamat K, Jawhari A, Alotibi S, Alzahrani K, AlMohimeed H, Alzahrani M et al. Relationship of self-esteem with depression, anxiety, and stress among dental and medical students in Jeddah, Saudi Arabia. J Int Med Dent. 2017; 4 (2): 61-68.

18. Johnson AK, Blackstone SR, Skelly A, Simmons W. The relationship between depression, anxiety, and burnout among physician assistant students: a multiinstitutional study. Heal Prof Educ [Internet]. 2020; 6 (3): 420-427. Available in: https://doi.org/10.1016/j. hpe.2020.04.003
19. Zancan BJ, De Lima DG, Lemos VSJ, Devos BEL, Andolhe $\mathrm{R}$, Bosi de Souza MTS. Depressive symptoms among healthcare undergraduate students. Rev Lat Am Enfermagem. 2020; 28: e3239.

20. Trindade Júnior SC, Sousa LFF de, Carreira LB. Generalized anxiety disorder and prevalence of suicide risk among medical students. Rev Bras Educ Med. 2027; 45 (2): 1-7.

21. Wenjun C, Ziwei F, Guogiang $H$, Mei H, Xinrong $X$, Jiaxin $D$ et al. The psychological impact of the COVID-19 epidemic on college students in China. Psychiatry Res [Internet]. 2020; 287: 1-5. Available in: from: http://www. embase.com/search/results?subaction=viewrecord\&fro m=export\&id=L2005406993\%0Ahttp://dx.doi.org/10.1016/j. psychres.2020.112934

22. Odriozola-Conzález P, Planchuelo-Gómez Á, Irurtia MJ, de Luis-García R. Psychological effects of the COVID-19 outbreak and lockdown among students and workers of a Spanish university. Psychiatry Res [Internet]. 2020; 290: 113108. Available from: https://doi.org/10.1016/j. psychres.2020.113108

23. Rahman ME, Zubayer AAl, Al Mazid Bhuiyan MR, Jobe MC, Ahsan Khan MK. Suicidal behaviors and suicide risk among Bangladeshi people during the COVID-19 pandemic: An online cross-sectional survey. Heliyon [Internet]. 2021; 7 (2): e05937. Available from: https://doi.org/10.1016/j.heliyon.2021. e05937

24. Grubic N, Badovinac S, Johri AM. Student mental health in the midst of the COVID-19 pandemic: a call for further research and immediate solutions. Int J Soc Psychiatry. 2020; 66 (5): 517-518.

25. Khan AH, Sultana MS, Hossain S, Hasan MT, Ahmed HU, Sikder MT. The impact of COVID-19 pandemic on mental health \& wellbeing among home-quarantined Bangladeshi students: a cross-sectional pilot study. J Affect Disord [Internet]. 2020; 277: 121-128. Available from: https:// doi.org/10.1016/j.jad.2020.07.135

26. Santana Campas MA, De Luna Velasco LE, Lozano Montes de Oca EE, Hermosillo de la Torre AE. Exploración del riesgo de suicidio en estudiantes universitarios mexicanos durante el aislamiento social por Covid-19. Rev Psicol la Univ Autónoma del Estado México. 2020; 9 (18): 54.

27. León OG, Montero I. A guide for naming research studies in Psychology. Int J Clin Heal Psychol. 2007; 7 (3): 847-862.

28. Plutchik R, van Praag HM, Conte HR, Picard S. Correlates of suicide and violence risk 1 : the suicide risk measure. Compr Psychiatry. 1989; 30 (4): 296-302.

29. Santana-Campas MA, Santoyo Telles F. Propiedades psicométricas de la escala riesgo suicida de Plutchik en una muestra de jóvenes mexicanos privados de la libertad. Av en Psicol. 2018; 26 (1): 57-64.

30. Daza P, Novy MD, Stanley AM, Averill P. The depression anxiety stress scale-21: spanish translation and validation 
with a hispanic sample. J Pof Psychopathol Behav Assess. 2002; 24 (3): 195-205.

31. Tijerina LZ, González GE, Gómez NM, Cisneros EMA, Rodríguez GKY, Ramos PEC. Depresión, ansiedad y estrés en estudiantes de nuevo ingreso a la educación superior. Rev Salud Pública Nutr. 2018; 17 (4): 41-47.

32. Quiñonez-Tapia F, Luévano TEM, Castañeda-Camey N. Confirmatory factor analysis and psychometric properties of the beck hopelessness scale in students in contexts of poverty in Mexico. Rev Psicopatol y Psicol Clin. 2019; 24 (2): 59-70.

33. Alamo PC, Baader MT, Antúnez SZ, Bagladi LV, Bejer TT. Beck hopelessness scale as a suicide risk screening tool among chilean university students. Rev Chil Neuropsiquiatr. 2019; 57 (2): 167-175.

34. Ellis P. Tamaños de efecto e interpretación de resultados de investigación en negocios internacionales. J Int Bus Stud. 2010; 41: 1581-1588.

35. Hair JF, Anderson RE, Tatham RL, Black WC. Análisis multivariante. 5ta edición. Madrid: Prentice Hall; 1999.

36. Análisis de mortalidad - Johns Hopkins Coronavirus Resource Center [Internet]. [Cited 2021 Oct 22]. Available in: https://coronavirus.jhu.edu/data/mortality

37. Palacios GR, Guerra AG, Sansores MA, Cómez S, Palacios GR, Guerra AG et al. Anxiety, depression and autonomic activity in nursing students, in the state of Guanajuato, Mexico. Ansiedad, depresión y actividad autonómica. 2020; 6: 81-103.

38. Luna D, Urquiza-Flores DI, Figuerola-Escoto RP, Carreño-Morales C, Meneses-Conzález F. Academic and sociodemographic predictors of anxiety and psychological well-being in mexican medical students. A cross-sectional study. Gac Med Mex. 2020; 156 (1): 40-46.

39. Saricali M, Satici SA, Satici B, Gocet-Tekin E, Griffiths MD. Fear of COVID-19, mindfulness, humor, and hopelessness: a multiple mediation analysis. Int J Ment Health Addict. 2020: 1-14.

40. Borda RN, Eyzaguirre RL. Autocontrol, preocupación, desesperanza y nivel socioeconómico en un contexto de pandemia por COVID19. Borda, Eyzaguirre, Ponce. Univ Católica Boliv "San Pablo" [Internet]. 2027; Ajayu. 19 (1): 5989. Available in: file:///C:/Users/usuario/OneDrive/Escritorio/ articulo 1.pdf

41. Muyor-Rodríguez J, Caravaca-Sánchez F, FernándezPrados JS. Covid-19 fear, resilience, social support, anxiety, and suicide among college students in Spain. Int J Environ Res Public Health. 2027; 18 (15): 8156.

42. Caballero-Domínguez CC, Jiménez-Villamizar MP, Campo-Arias A. Suicide risk during the lockdown due to coronavirus disease (COVID-19) in Colombia. Death Stud [Internet]. 2020; 1-6. Available in: https://doi.org/10.1080/07 481187.2020.1784312

43. Hermosillo-De-la-torre AE, Arteaga-De-luna SM, AcevedoRojas DL, Juárez-Loya A, Jiménez-Tapia JA, PedrozaCabrera FJ et al. Psychosocial correlates of suicidal behavior among adolescents under confinement due to the COVID-19 pandemic in Aguascalientes, Mexico: A cross-sectional population survey. Int J Environ Res Public Health. 2027; 18 (9): 1-17. 